PROCEEDINGS OF THE

AMERICAN MATHEMATICAL SOCIETY

Volume 128, Number 11, Pages 3335-3340

S 0002-9939(00)05435-6

Article electronically published on May 11, 2000

\title{
ON THE WEAK LIMIT OF MAPPINGS WITH FINITE DISTORTION
}

\author{
BAISHENG YAN
}

(Communicated by Albert Baernstein II)

\begin{abstract}
We give a new proof that the limit of a weakly convergent sequence of mappings with finite distortion also has finite distortion. The result has been recently proved by Gehring and Iwaniec using the biting convergence of Jacobians. We present a different proof using simply the lower semi-continuity of quasiconvex functionals.
\end{abstract}

\section{INTRODUCTION}

Let $f: \Omega \rightarrow \mathbf{R}^{n}$ be a mapping in the Sobolev space $W_{l o c}^{1, p}\left(\Omega ; \mathbf{R}^{n}\right)$, where $\Omega$ is a domain in $\mathbf{R}^{n}$. Then the Jacobian matrix $D f(x)$ and its determinant $J(x, f)=$ $\operatorname{det} D f(x)$ are well-defined at almost every point $x \in \Omega$. We shall use $M^{n \times n}$ to denote the space of all $n \times n$ real matrices equipped with the operator norm

$$
|\xi|=\max \{|\xi v||| v \mid=1\} .
$$

Definition 1.1. A mapping $f \in W_{l o c}^{1, n}\left(\Omega ; \mathbf{R}^{n}\right)$ is said to have finite distortion if there exists a finite measurable function $K(x) \geq 0$ such that

$$
|D f(x)|^{n} \leq K(x) J(x, f)
$$

for almost every $x \in \Omega$.

From this definition, a mapping $f$ with finite distortion has the property that either the Jacobian matrix $D f(x)=0$ or its determinant $J(x, f)>0$; in the latter case the matrix $D f(x)$ is invertible.

Definition 1.2. The outer and inner dilatation functions $K_{O}(x, f)$ and $K_{I}(x, f)$ of a mapping $f$ with finite distortion are defined as follows:

$$
\left\{\begin{array}{l}
K_{O}(x, f)=K_{I}(x, f)=1 \quad \text { if } D f(x)=0 ; \\
K_{O}(x, f)=K_{O}(D f(x)), \\
K_{I}(x, f)=K_{I}(D f(x)) \quad \text { if } J(x, f)>0,
\end{array}\right.
$$

where, for any invertible matrices $\xi$,

$$
K_{O}(\xi)=|\xi|^{n} / \operatorname{det} \xi, \quad K_{I}(\xi)=K_{O}\left(\xi^{-1}\right) .
$$

We shall prove the following theorem; see also Gehring and Iwaniec [3].

Received by the editors September 17, 1998 and, in revised form, January 11, 1999.

1991 Mathematics Subject Classification. Primary 30C65, 30C70, 49 J45.

(C)2000 American Mathematical Society 
Theorem 1.3. Let $f_{\nu}: \Omega \rightarrow \mathbf{R}^{n}$ be a sequence of mappings with finite distortion which converges weakly in $W_{\text {loc }}^{1, n}\left(\Omega ; \mathbf{R}^{n}\right)$ to a mapping $f$. Suppose there exists a finite measurable function $M(x)$ such that

$$
K\left(x, f_{\nu}\right) \leq M(x)<\infty
$$

almost everywhere in $\Omega$ for all $\nu=1,2, \ldots$, where $K(x, f)$ is either $K_{O}(x, f)$ or $K_{I}(x, f)$. Then the limit mapping $f$ has finite distortion. Moreover, for any subsequence $f_{\nu_{k}}$, one has

$$
K_{O}(x, f) \leq \limsup _{k \rightarrow \infty} K_{O}\left(x, f_{\nu_{k}}\right)
$$

and

$$
K_{I}(x, f) \leq \limsup _{k \rightarrow \infty} K_{I}\left(x, f_{\nu_{k}}\right)
$$

for almost every $x \in \Omega$.

This theorem is a refinement of Reshetnyak's convergence theorem [6], Theorem $9.2]$ concerning mappings of bounded distortion, that is, mappings $f$ with $K(x) \leq$ $K<\infty$ for a constant $K$ in (1.1). For such mappings, the (maximum) outer and inner dilatations are defined by

$$
K_{O}(f)=\left\|K_{O}(x, f)\right\|_{L^{\infty}(\Omega)}, \quad K_{I}(f)=\left\|K_{I}(x, f)\right\|_{L^{\infty}(\Omega)} .
$$

In Theorem 1.3. if $f_{\nu}$ has bounded distortion and satisfies

$$
K_{O}\left(f_{\nu}\right) \leq M<\infty
$$

for a constant $M$ and all $\nu=1,2, \ldots$, then from (1.4) and (1.5) we can establish

$$
K_{O}(f) \leq \liminf _{\nu \rightarrow \infty} K_{O}\left(f_{\nu}\right), \quad K_{I}(f) \leq \liminf _{\nu \rightarrow \infty} K_{I}\left(f_{\nu}\right),
$$

which recovers Reshetnyak's convergence theorem [6], Theorem 9.2].

Finally, we remark that the estimate (1.4) seems weaker than the estimate given in Gehring and Iwaniec [3 Remark 1.7] in terms of biting convergence. We also refer to [3, 4] for the convergence results regarding other dilatation functions.

\section{VARIATIONAL APPROACHES}

In order to present our proof of Theorem 1.3, we need some variational characterizations of mappings with finite distortion.

For any given finite measurable function $K(x)$ on $\Omega$, consider the function from $\Omega \times M^{n \times n}$ to $\mathbf{R}$ defined by

$$
F_{1}(x, \xi)=\max \left\{0,|\xi|^{n}-K(x) \operatorname{det} \xi\right\} .
$$

It is easy to see that

$$
0 \leq F_{1}(x, \xi) \leq \rho_{1}(x)|\xi|^{n},
$$

where $\rho_{1}(x)=1+|K(x)|$, and the condition (1.1) in Definition 1.1 is equivalent to

$$
F_{1}(x, D f(x))=0
$$

for almost every $x \in \Omega$. Hence, any mapping $f$ with finite distortion is an absolute minimizer of the functional

$$
I_{1}(u, \Omega)=\int_{\Omega} F_{1}(x, D u(x)) d x
$$

where $F_{1}(x, \xi)$ is defined by (2.1) with $K(x)=K_{O}(x, f)$. 
In order to give another characterization using the inner dilatation function $K_{I}(x, f)$, we need some notation. Let $\xi^{\#}$ be the matrix of cofactors of matrix $\xi$ such that

$$
\xi \xi^{\#}=\xi^{\#} \xi=(\operatorname{det} \xi) I
$$

where $I$ is the identity matrix. For invertible matrices $\xi$, this shows that $\xi^{-1}=$

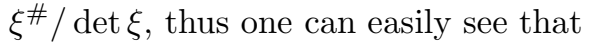

$$
K_{I}(\xi)=\left|\xi^{\#}\right|^{n} /(\operatorname{det} \xi)^{n-1} .
$$

Now let $P(x)$ be any finite measurable function on $\Omega$. Consider

$$
F_{2}(x, \xi)=\max \left\{0,\left|\xi^{\#}\right|^{\frac{n}{n-1}}-P(x) \operatorname{det} \xi\right\} .
$$

Then $F_{2}(x, \xi)$ also satisfies

$$
0 \leq F_{2}(x, \xi) \leq \rho_{2}(x)|\xi|^{n},
$$

where $\rho_{2}(x)=c_{n}+|P(x)|$. If $f$ is a mapping with finite distortion, then

$$
F_{2}(x, D f(x))=0
$$

for almost every $x \in \Omega$, where $F_{2}(x, \xi)$ is defined as above with $P(x)=K_{I}(x, f)^{1 / n-1}$. Therefore $f$ is an absolute minimizer of the functional

$$
I_{2}(u, \Omega)=\int_{\Omega} F_{2}(x, D u(x)) d x .
$$

Finally, we remark that functions $F_{1}, F_{2}$ defined above have the important property of quasiconvexity introduced by Morrey [5]; see also Ball [2].

Proposition 2.1. Let $F(x, \xi)$ denote one of $F_{1}(x, \xi)$ and $F_{2}(x, \xi)$ defined by (2.1) and (2.3). Then, for almost every $x \in \Omega, F(x, \xi)$ is quasiconvex in $\xi$ in the sense that

$$
F(x, \xi) \leq \frac{1}{|\Omega|} \int_{\Omega} F(x, \xi+D \phi(y)) d y, \quad \forall \phi \in C_{0}^{\infty}\left(\Omega ; \mathbf{R}^{n}\right)
$$

Proof. For any $\xi \in M^{n \times n}, \phi \in C_{0}^{\infty}\left(\Omega ; \mathbf{R}^{n}\right)$, it follows that [2, 5]

$$
\operatorname{det} \xi=\frac{1}{|\Omega|} \int_{\Omega} \operatorname{det}(\xi+D \phi(y)) d y, \quad \xi^{\#}=\frac{1}{|\Omega|} \int_{\Omega}(\xi+D \phi(y))^{\#} d y .
$$

Then, (2.4) follows from the definition of $F_{1}, F_{2}$ and Jensen's inequality.

\section{LOWER SEMICONTINUITY}

In what follows, we assume that $F(x, \xi)$ is a Carathéodory function from $\Omega \times$ $M^{n \times n}$ to $\mathbf{R}$ in the sense that $F(x, \xi)$ is continuous in $\xi$ for almost every $x \in \Omega$ and measurable in $x$ for all $\xi \in M^{n \times n}$. Assume also that there exists a finite measurable function $\rho(x) \geq 0$ such that

$$
0 \leq F(x, \xi) \leq \rho(x)|\xi|^{p}
$$

for almost every $x \in \Omega$, where $1 \leq p<\infty$ is a constant. We need the following lower semicontinuity theorem mainly due to Acerbi and Fusco [1]. 
Theorem 3.1. Let $F(x, \xi)$ be given as above. Suppose for almost every $x \in \Omega$ the function $F(x, \xi)$ is quasiconvex in $\xi$ in the sense as defined in Proposition 2.1. For any measurable subset $E$ of $\Omega$ and $t>0$ let

$$
E_{t}=\{x \in E \mid \rho(x)<t\} .
$$

If $f_{\nu}: \Omega \rightarrow \mathbf{R}^{n}$ is a sequence of mappings which converges weakly in $W^{1, p}\left(\Omega ; \mathbf{R}^{n}\right)$ to a mapping $f$, then, for every $t>0$, one has

$$
\int_{E_{t}} F(x, D f(x)) d x \leq \liminf _{\nu \rightarrow \infty} \int_{E_{t}} F\left(x, D f_{\nu}(x)\right) d x .
$$

Proof. Consider $G(x, \xi)=F(x, \xi) \chi_{E_{t}}(x)$, where $\chi_{S}$ denotes the characteristic function of set $S$. Then $G(x, \xi)$ is a Carathéodory function and, for almost every $x \in \Omega$, $G(x, \xi)$ is quasiconvex in $\xi$ and satisfies

$$
0 \leq G(x, \xi) \leq t|\xi|^{p} .
$$

Therefore, by the lower semicontinuity theorem of Acerbi and Fusco 11, Theorem II.4], the functional $J(u)=\int_{\Omega} G(x, D u)$ is (sequentially) weakly lower semicontinuous on $W^{1, p}\left(\Omega ; \mathbf{R}^{n}\right)$. Hence,

$$
\int_{E_{t}} F(x, D f)=J(f) \leq \liminf _{\nu \rightarrow \infty} J\left(f_{\nu}\right)=\liminf _{\nu \rightarrow \infty} \int_{E_{t}} F\left(x, D f_{\nu}\right) .
$$

The theorem is proved.

Theorem 3.2. Suppose a sequence of mappings $f_{\nu}: \Omega \rightarrow \mathbf{R}^{n}$ converges weakly in $W_{\text {loc }}^{1, n}\left(\Omega ; \mathbf{R}^{n}\right)$ to a mapping $f$. Let $K(x)$ and $P(x)$ be any given finite measurable functions in $\Omega$, and let $F_{1}(x, \xi)$ and $F_{2}(x, \xi)$ be defined by (2.1) and (2.3), respectively. Assume $F(x, \xi)$ is one of $F_{1}$ and $F_{2}$. Let $E$ be a measurable subset of $\Omega$ such that

$$
\lim _{\nu \rightarrow \infty} \int_{E} F\left(x, D f_{\nu}(x)\right) d x=0 .
$$

Then $F(x, D f(x))=0$ for almost every $x \in E$.

Proof. The theorem follows easily from Proposition 2.1 and Theorem 3.1.

Finally, we prove a result which enables us to estimate one dilatation function in terms of the other.

Lemma 3.3. Let $f \in W_{l o c}^{1, n}\left(\Omega ; \mathbf{R}^{n}\right)$ be a mapping with finite distortion. Then

$$
K_{O}(x, f) \leq K_{I}(x, f)^{n-1}, \quad K_{I}(x, f) \leq K_{O}(x, f)^{n-1}
$$

for almost every $x \in \Omega$.

Proof. Note that the functions $K_{O}(\xi)$ and $K_{I}(\xi)$ defined by (1.3) can be represented by the principal values of $\xi$, that is, the eigenvalues of the matrix $\sqrt{\xi^{T} \xi}$. Let $\operatorname{det} \xi \neq 0$ and let $0<\lambda_{1} \leq \lambda_{2} \leq \cdots \leq \lambda_{n}$ be the principal values of $\xi$. Then it is easy to see that

$$
K_{O}(\xi)=\lambda_{n}^{n} / \lambda_{1} \lambda_{2} \cdots \lambda_{n}, \quad K_{I}(\xi)=\lambda_{1} \lambda_{2} \cdots \lambda_{n} / \lambda_{1}^{n} .
$$

Therefore,

$$
K_{O}(\xi) \leq K_{I}(\xi)^{n-1}, \quad K_{I}(\xi) \leq K_{O}(\xi)^{n-1},
$$

and the lemma follows from the definition of $K_{O}(x, f)$ and $K_{I}(x, f)$. 


\section{Proof of Theorem 1.3}

By Lemma 3.3, we can assume that the function $K(x, f)$ in Theorem 1.3 is $K_{O}(x, f)$. Let $F_{1}(x, \xi)$ be defined by (2.1) with $K(x)=M(x)$ as given in the theorem. We then have $F_{1}\left(x, D f_{\nu}(x)\right)=0$. Thus by Theorem 3.2 it follows that $F_{1}(x, D f(x))=0$, that is,

$$
|D f(x)|^{n} \leq M(x) J(x, f)
$$

for almost every $x \in \Omega$. Therefore, $f$ is a mapping with finite distortion and $K_{O}(x, f) \leq M(x)$ for almost every $x \in \Omega$.

We need to show estimates (1.4) and (1.5). For this purpose, we may assume that the subsequence $f_{\nu_{k}}$ is the original full sequence $f_{\nu}$. Let

$$
L(x)=\limsup _{\nu \rightarrow \infty} K_{O}\left(x, f_{\nu}\right), \quad Q(x)=\limsup _{\nu \rightarrow \infty} K_{I}\left(x, f_{\nu}\right) .
$$

We need to show

$$
K_{O}(x, f) \leq L(x), \quad K_{I}(x, f) \leq Q(x)
$$

for almost every $x \in \Omega$.

If $J(x, f)=0$, that is, $D f(x)=0$, then $K_{O}(x, f)=1 \leq L(x)$ and $K_{I}(x, f)=$ $1 \leq Q(x)$, and hence (4.1) holds. So we have only to prove (4.1) for almost every $x$ in the measurable set $\Omega^{\prime}=\{x \in \Omega \mid J(x, f)>0\}$. We assume $\left|\Omega^{\prime}\right|>0$, and let

$$
N=\left\{x \in \Omega^{\prime} \mid L(x)<K_{O}(x, f)\right\}, \quad R=\left\{x \in \Omega^{\prime} \mid Q(x)<K_{I}(x, f)\right\} .
$$

We need to show $|N|=|R|=0$. Note that

$$
N=\bigcup_{m=1}^{\infty} \bigcup_{k=1}^{\infty} N_{m k}, \quad R=\bigcup_{m=1}^{\infty} \bigcup_{k=1}^{\infty} R_{m k},
$$

where

$$
N_{m k}=\bigcap_{\nu=k}^{\infty}\left\{x \in \Omega^{\prime} \mid K_{O}\left(x, f_{\nu}\right) \leq K_{O}(x, f)-\frac{1}{m}\right\}
$$

and

$$
R_{m k}=\bigcap_{\nu=k}^{\infty}\left\{x \in \Omega^{\prime} \mid K_{I}\left(x, f_{\nu}\right) \leq K_{I}(x, f)-\frac{1}{m}\right\} .
$$

We shall prove $\left|N_{m k}\right|=\left|R_{m k}\right|=0$ for all $m, k=1,2, \ldots$ and hence $|N|=|R|=0$. This will complete the proof of (4.1).

Proof of $\left|N_{m k}\right|=0$. Note that $K_{O}\left(x, f_{\nu}\right) \leq K_{O}(x, f)-1 / m$ for all $x \in N_{m k}$ and all $\nu \geq k$. We have

$$
\left|D f_{\nu}(x)\right|^{n} \leq\left(K_{O}(x, f)-\frac{1}{m}\right) J\left(x, f_{\nu}\right)
$$

for all $\nu=k, k+1, \ldots$ and almost every $x \in E=N_{m k}$. Hence, by Theorem 3.2,

$$
|D f(x)|^{n} \leq\left(K_{O}(x, f)-\frac{1}{m}\right) J(x, f)
$$

for almost every $x \in E=N_{m k}$. Since $J(x, f)>0$ for $x \in E$, it follows that $K_{O}(x, f) \leq K_{O}(x, f)-1 / m$ for almost every $x \in E$. Hence, $|E|=\left|N_{m k}\right|=0$ for all $m, k=1,2, \ldots ;$ the proof is complete. 
Proof of $\left|R_{m k}\right|=0$. This is similar to the previous one. Note that $K_{I}\left(x, f_{\nu}\right) \leq$ $K_{I}(x, f)-1 / m$ for all $x \in R_{m k}$ and all $\nu \geq k$. Let $F_{2}(x, \xi)$ be the function defined by (2.3) with

$$
P(x)=\left(K_{I}(x, f)-1 / m\right)^{\frac{1}{n-1}} .
$$

We then have $F_{2}\left(x, D f_{\nu}(x)\right)=0$ for almost every $x \in E=R_{m k}$ and all $\nu \geq k$. Therefore, again by Theorem $3.2, F_{2}(x, D f(x))=0$ for almost every $x \in R_{m k}$. This implies

$$
\left|(D f(x))^{\#}\right|^{\frac{n}{n-1}} \leq P(x) J(x, f)
$$

and thus by (2.2)

$$
K_{I}(x, f) \leq(P(x))^{n-1}=K_{I}(x, f)-1 / m
$$

for almost every $x \in R_{m k}$. Thus $\left|R_{m k}\right|=0$. The proof of Theorem 1.3 is complete.

\section{ACKNOWLEDGMENTS}

The author would like to thank the referee for helpful comments.

\section{REFERENCES}

[1] E. Acerbi and N. Fusco, Semicontinuity problems in the calculus of variations, Arch. Rational Mech. Anal., 86 (1984), 125-145. MR 85m:49021

[2] J. M. Ball, Convexity conditions and existence theorems in nonlinear elasticity, Arch. Rational Mech. Anal., 63 (1977), 337-403. MR 57:14788

[3] F. Gehring and T. Iwaniec, The limit of mappings with finite distortion, Ann. Acad. Sci. Fenn. Math., 24 (1999), 253-264. MR 99m:30041

[4] T. Iwaniec, The failure of lower semicontinuity for the linear dilatation, Bull. London Math. Soc., 30 (1998), 55-61. MR 98i:30033

[5] C. B. Morrey, "Multiple Integrals in the Calculus of Variations," Springer-Verlag, Berlin, Heidelberg, New York, 1966. MR 34:2380

[6] Yu. G. Reshetnyak, "Space Mappings with Bounded Distortion," Transl. Math. Mono., Amer. Math. Soc., Vol. 73, 1989. MR 90d:30067 48824

Department of Mathematics, Michigan State University, East Lansing, Michigan

E-mail address: yan@math.msu.edu 